CERN-TH. 7294/94

MPI-PhT/94-32

hep-ph/9406424

\title{
Radiative B-decay as a test of CKM unitarity
}

\author{
Gautam Bhattacharyya ${ }^{()}$and Gustavo C. Branco *) *) \\ Theory Division, CERN, \\ CH 1211, Geneva 23, SWITZERLAND \\ and \\ Debajyoti Choudhury $\diamond$ \\ Max-Planck-Institut für Physik - Werner Heisenberg Institut, \\ Föhringer Ring 6, D-80805 Munich, GERMANY.
}

\begin{abstract}
We point out that $R \equiv \operatorname{Br}(b \rightarrow d \gamma) / B r(b \rightarrow s \gamma)$ is a sensitive probe of possible violation of CKM unitarity. We compute $R$ in a minimal extension of the Standard Model containing an additional isosinglet charge $(-1 / 3)$ quark, which leads to a deviation from CKM unitarity.
\end{abstract}

CERN-TH. 7294/94

June 1994

๑) gautam@cernvm.cern.ch

\&) gbranco@cernvm.cern.ch

») debchou@iws186.mppmu.mpg.de

*) On leave of absence from: Departamento de Fisica and CFIF/UTL, Instituto Superior Técnico, Avenida Rovisco Pais, 1096 Lisboa Cedex, Portugal. 
The study of radiative $B$-decays provides an important test of the Standard Model $(\mathrm{SM})$ and is indeed a sensitive probe of physics beyond the SM. The recent discovery, by the CLEO collaboration, [1] of the decay $B \rightarrow K^{*} \gamma$ with a branching ratio $\operatorname{Br}\left(B \rightarrow K^{*} \gamma\right)=(4.5 \pm 1.5 \pm 0.9) \times 10^{-5}$ provides further motivation for this study. Within the framework of the SM, the rare decays $b \rightarrow d \gamma$ and $b \rightarrow s \gamma$ provide independent measurements of the Cabibbo-Kobayashi-Maskawa (CKM) elements $V_{t d}$ and $V_{t s}$, respectively, which can be tested against the ones measured from $B_{d^{-}} \bar{B}_{d}$ and $B_{s^{-}} \bar{B}_{s}$ mixings [2].

In this note we will point out that the decay amplitudes $b \rightarrow q \gamma(q=d, s)$ have a crucial dependence on the unitarity of the $(3 \times 3)$ CKM matrix. Thus the magnitude of such rare decays provides an excellent testing ground for new physics leading to the violation of the CKM unitarity. For definiteness, we will analyse the above radiative decays in the framework of a minimal extension of the SM where a charge $(-1 / 3) S U(2)$ singlet quark is introduced. Although we treat this extension beyond the SM from a phenomenological viewpoint, there are theoretically appealing motivations behind its consideration, including $E_{6}$ grand-unified theories and some superstring-inspired models [3]. Furthermore, isosinglet quarks provide a simple solution [4] to the strong CP problem [5]. We will show that the branching fraction $b \rightarrow d \gamma$ can change significantly due to lack of GIM cancellation resulting from unitarity violation in the new CKM matrix. However, the branching fraction $b \rightarrow s \gamma$ is not affected to any level of significance. Therefore, the most important impact of the violation of CKM unitarity would be a change in the ratio $R \equiv B r(b \rightarrow d \gamma) / B r(b \rightarrow s \gamma)$ which, in the SM, provides a reliable measure of $\left|V_{t d} / V_{t s}\right|$.

Before describing the model in some detail, let us first recall the general structure of the radiative $b$-decay and fix our notation. To the leading order, the quark-level branching ratio $b \rightarrow q \gamma(q=d, s)$ is given in units of the semileptonic $b$-decay branching ratio, as

$$
\frac{B r(b \rightarrow q \gamma)}{B r(b \rightarrow c e \bar{\nu})}=\frac{6 \alpha}{\pi \rho \lambda}\left|\frac{V_{t b} V_{t q}^{*}}{V_{c b}}\right|^{2}\left[\eta^{16 / 23} A_{\gamma}+\frac{8}{3}\left(\eta^{14 / 23}-\eta^{16 / 23}\right) A_{g}+C\right]^{2}
$$

where $\eta \equiv \alpha_{S}\left(M_{Z}\right) / \alpha_{S}\left(m_{b}\right)=0.548, \rho=\left(1-8 r^{2}+8 r^{6}-r^{8}-24 r^{4} \ln r\right)$ with $r=m_{c} / m_{b}$, $\lambda=1-1.61 \alpha_{S}\left(m_{b}\right) / \pi$, and $C(=-0.1766)$ is a term emanating from a complete calculation of the leading-logarithmic QCD corrections [6]; $V$ is the standard CKM matrix. It may be noted that the $m_{b}{ }^{5}$ dependence in the partial decay widths of the $b$ quark cancels out in eq. (11). An $\mathcal{O}\left(m_{q}^{2} / m_{b}^{2}\right)$ part in the branching ratio has been neglected. We use $\operatorname{Br}(b \rightarrow c e \bar{\nu})=0.107$. $A_{\gamma}$ and $A_{g}$ are the coefficients of the effective operators for the magnetic and chromomagnetic moment couplings for $b \rightarrow q$ 
transitions [2] following from

$$
\mathcal{L}_{e f f}=\sqrt{\frac{G_{F}^{2}}{8 \pi^{3}}} V_{t b} V_{t q}^{*} \bar{q} \sigma^{\mu \nu}\left[\sqrt{\alpha} A_{\gamma} F_{\mu \nu}+\sqrt{\alpha_{S}} A_{g} T_{a} G_{\mu \nu}^{a}\right]\left(m_{b} P_{R}+m_{q} P_{L}\right) b,
$$

where $P_{R, L}=\left(1 \pm \gamma_{5}\right) / 2$. Within the framework of the SM, the effective operator in eq. (2), to lowest order, is obtained from the 'penguin' diagrams in fig. 1a. For the sake of making this note self-contained, we give the explicit expressions of $A_{\gamma}$ and $A_{g}$ :

$$
A_{\gamma}=x\left[\frac{7-5 x-8 x^{2}}{24(x-1)^{3}}+\frac{x(3 x-2)}{4(x-1)^{4}} \ln x\right]
$$

and

$$
A_{g}=x\left[\frac{2+5 x-x^{2}}{8(x-1)^{3}}-\frac{3 x}{4(x-1)^{4}} \ln x\right]
$$

where $x=m_{t}^{2} / M_{W}^{2}$. In all our discussions we shall assume that $\frac{m_{c}^{2}}{M_{W}^{2}} \simeq 0$.

It may be noted that the derivations of eqs. (3) and (4) depend crucially on the validity of CKM unitarity. For the sake of illustration, particularly since we deal with a model that violates this unitarity, we present the contribution $f_{i}^{W}$ that replaces $A_{\gamma}$ or $A_{g}$ in eq. (2) for each individual diagram of fig. 1a:

$$
\begin{aligned}
f_{1}^{W} & =\frac{1}{4} \bar{\xi}_{1}(x)+\frac{1}{2} \bar{\xi}_{2}(x) \\
f_{2}^{W} & =x\left[\frac{1}{2} \bar{\xi}_{0}(x)-\frac{3}{4} \bar{\xi}_{1}(x)+\frac{1}{4} \bar{\xi}_{2}(x)\right] \\
f_{3}^{W} & +f_{4}^{W}=\frac{1}{4} \bar{\xi}_{1}(x) \\
f_{5}^{W} & =Q_{t}\left[\xi_{0}(x)-\frac{3}{2} \xi_{1}(x)+\frac{1}{2} \xi_{2}(x)\right] \\
f_{6}^{W} & =\frac{Q_{t}}{4} x\left[\xi_{1}(x)+\xi_{2}(x)\right],
\end{aligned}
$$

where $Q_{t}$ is the charge of the top-quark and

$$
\begin{aligned}
\xi_{n}(x) & =\int_{0}^{1} \frac{z^{n+1} \mathrm{~d} z}{1+(x-1) z}=\frac{-1}{(1-x)^{n+2}}\left[\ln x+\sum_{k=1}^{n+1}(-1)^{k}\left(\begin{array}{c}
n+1 \\
k
\end{array}\right) \frac{x^{k}-1}{k}\right] \\
\bar{\xi}_{n}(x) & =\frac{1}{x} \xi_{n}\left(\frac{1}{x}\right) .
\end{aligned}
$$

In the event of a unitary $V^{C K M}$, GIM cancellation ensures that we need consider only

$$
\begin{aligned}
\sum_{j=1}^{6}\left\{f_{j}^{W}(x)-f_{j}^{W}(0)\right\} & =A_{\gamma}, \\
\frac{1}{Q_{t}} \sum_{j=5}^{6}\left\{f_{j}^{W}(x)-f_{j}^{W}(0)\right\} & =A_{g},
\end{aligned}
$$


leading to the same results as in eqs. (3) and (4) respectively.

Now we briefly describe our model and the essential modifications from the SM. We introduce an extra down-type quark $(D)$, whose left- and right-handed components are both $S U(2)$ singlets. Consequently, the CKM matrix $V^{C K M}$ is a $(3 \times 4)$ one. Without loss of generality we can assume that the up-quark mass matrix is diagonal. $V^{C K M}$ then consists of the first three lines of the $(4 \times 4)$ unitary matrix $W$ which relates the left-handed components of the down-quark weak and mass eigenstates:

$$
\left(\begin{array}{c}
d_{i}^{\circ} \\
D^{\circ}
\end{array}\right)_{L}=W\left(\begin{array}{c}
d_{i} \\
D
\end{array}\right)_{L}
$$

where $i=1,2,3$ and the weak eigenstates are denoted by the superscript 0 . It can readily be seen [7] that whereas

$$
\left(V V^{\dagger}\right)_{i j}=\delta_{i j}, \quad z_{\alpha \beta} \equiv\left(V^{\dagger} V\right)_{\alpha \beta} \neq \delta_{\alpha \beta} \quad\left(V \equiv V^{C K M}\right) .
$$

The weak gauge currents can be expressed in terms of the mass eigenstates as

$$
\begin{aligned}
J_{\mu}^{W} & =\frac{g}{\sqrt{2}} \bar{u}_{L i} V_{i \alpha}^{C K M} \gamma_{\mu} d_{L \alpha} \\
J_{\mu}^{Z} & =\frac{g}{\cos \theta_{W}}\left[t_{3}^{u} \bar{u}_{L i} \gamma_{\mu} u_{L i}+z_{\alpha \beta} t_{3}^{d} \bar{d}_{L \alpha} \gamma_{\mu} d_{L \beta}-\sin ^{2} \theta_{W} J_{\mu}^{e m}\right]
\end{aligned}
$$

where $i=1,2,3 ; \alpha, \beta=1,2,3,4$ and $t_{3}^{u}\left(t_{3}^{d}\right)=1 / 2(-1 / 2)$. The generic symbols $z_{\alpha \beta}$ then parametrise the tree-level flavour dependence of the down-sector neutral currents.

Within the SM sector the couplings $z_{\alpha \beta}$ (for $\alpha \neq \beta$ ) are naturally suppressed by the ratio of the standard quark masses to the vector-like quark masses (see ref. [7] for details) and are strongly constrained by experiments. Of special interest to us are the couplings $z_{b s}$ and $z_{b d}$, which are constrained by $\operatorname{Br}\left(B \rightarrow X \mu^{+} \mu^{-}\right) \leq 5.0 \times 10^{-5}$ measured by the UA1 Collaboration [8], leading to the bounds [7]:

$$
\left|\frac{z_{b d}}{V_{c b}}\right| \leq 0.029, \quad\left|\frac{z_{b s}}{V_{c b}}\right| \leq 0.029
$$

For convenience, we write the explicit expressions of $z_{b d}$ and $z_{b s}$ as parts of the unitarity quadrangles:

$$
\begin{aligned}
& z_{b d}=V_{t b} V_{t d}^{*}+V_{c b} V_{c d}^{*}+V_{u b} V_{u d}^{*} \\
& z_{b s}=V_{t b} V_{t s}^{*}+V_{c b} V_{c s}^{*}+V_{u b} V_{u s}^{*}
\end{aligned}
$$

So far we have discussed only the $W$ - and $Z$-mediated interactions. The reason why we have not emphasized the Higgs contributions is due to the fact that they depend 
on the Higgs structure we choose for the specific model with an isosinglet quark. The minimal structure is, of course, having only one Higgs doublet as in the SM with a bare mass term for the isosinglet. In this case, we have checked that the contributions from the Higgs-mediated penguins are small with respect to the dominant contribution to $b \rightarrow d \gamma$, which arises from $W$ - and $Z$-mediated penguins.

Armed with the above information, let us now look at the diagrams of interest to us. For simplicity, we assume that $z_{b s}=0$, so that the violation of CKM unitarity is all contained in $z_{b d}$. (We also assume that $z_{d s}=0$ in view of the extremely tight restriction from $K-\bar{K}$ mixing.) Note that the inclusion of the isosinglet leaves unaffected the apparent structure of the individual diagrams in fig. 1a. A crucial difference, however, is brought about by the lack of GIM suppression. The flavour independent terms no longer cancel; rather, they lead to contributions proportional to $z_{b d}$. Unitarity violation also shows up through a new set of $Z$-mediated penguins (fig. 1b), which originate as a result of tree-level flavour mixing at the $Z$-vertex. The contributions $f_{i}^{Z}$ from the diagrams of fig. $1 \mathrm{~b}$, analogous to the $f_{1}^{W}$ of fig. $1 \mathrm{a}$, are given by $\left(y=m_{d, b}^{2} / M_{Z}^{2} \simeq\right.$ $\left.0, y_{D}=m_{D}^{2} / M_{Z}^{2}\right)$ :

$$
\begin{aligned}
f_{1}^{Z}+f_{2}^{Z} & =Q_{d} t_{3}^{d}\left[a_{L}^{d}\left\{4 \xi_{0}(y)-6 \xi_{1}(y)+2 \xi_{2}(y)\right\}-4 a_{R}^{d}\left\{\xi_{0}(y)-\xi_{1}(y)\right\}\right], \\
f_{3}^{Z} & =Q_{d}\left(t_{3}^{d}\right)^{2}\left[2 \xi_{0}\left(y_{D}\right)-3 \xi_{1}\left(y_{D}\right)+\xi_{2}\left(y_{D}\right)\right],
\end{aligned}
$$

where $a_{L}^{d}=t_{3}^{d}-Q_{d} \sin ^{2} \theta_{W}, a_{R}^{d}=-Q_{d} \sin ^{2} \theta_{W}$, and the integrals $\xi_{i}$ are listed in eq. (6). As a result, the expressions of $A_{\gamma}$ and $A_{g}$ are modified significantly to

$$
\begin{aligned}
& A_{\gamma} \longrightarrow A_{\gamma}+\left(\frac{z_{b d}}{V_{t b} V_{t d}^{*}}\right)^{I S}\left(c_{\gamma}^{W}+c_{\gamma}^{Z}\right), \\
& A_{g} \longrightarrow A_{g}+\left(\frac{z_{b d}}{V_{t b} V_{t d}^{*}}\right)^{I S}\left(c_{g}^{W}+c_{g}^{Z}\right) .
\end{aligned}
$$

Above, the supercript $(I S)$ refers to the CKM elements in the presence of an isosinglet quark; $c_{\gamma, g}^{W, Z}$ are constants arising from the $W(Z)$-mediated photon(gluon)-penguins. These numbers can easily be derived from eqs. (5),(16),(13) to be

$$
\begin{aligned}
c_{\gamma}^{W} & \simeq \sum_{j=1}^{6} f_{j}^{W}(0)=23 / 36, \\
c_{g}^{W} & \simeq \frac{1}{Q_{t}} \sum_{j=5}^{6} f_{j}^{W}(0)=1 / 3, \\
c_{\gamma}^{Z} & \simeq \sum_{j=1}^{2} f_{j}^{Z}(0)=-13 / 108, \\
c_{g}^{Z} & \simeq \frac{1}{Q_{d}} \sum_{j=1}^{2} f_{j}^{Z}(0)=13 / 36 .
\end{aligned}
$$


Needless to say, one should also add the contribution of $f_{3}^{Z}$ to the above. This obviously depends on $m_{D}$ and thus introduces an additional unknown. However, it can easily be checked that such contributions are typically smaller and make little quantitative impact. For example, even for relatively large $z_{i 4}$ consistent with the unitarity of $W$, these extra contributions, which we neglect, are $(-1 / 60)$ to $c_{\gamma}^{Z}$ and $(1 / 20)$ to $c_{g}^{Z}$ for $y_{D}=2$ and are even smaller for larger values of $y_{D}$.

The next task is to determine the element $V_{t d}^{I S}$. It ought to be stressed that the experimentally allowed range of values of the CKM matrix elements in this scenario differ from the corresponding ones in the SM. For example, in the SM, the element $V_{t d}$ is determined by comparing the $B_{d}-\bar{B}_{d}$ mixing data with its prediction driven by the $t$ mediated box. In this scenario, the extraction of $V_{t d}$ is more complicated, though, since $B_{d}-\bar{B}_{d}$ mixing receives a tree-level contribution due to flavour-violating $Z$ couplings in the down-sector. The bound of eq. (11) allows for this tree level contribution to be comparable with, or even dominate, the $t$-mediated SM box. In the following, we will neglect the effect of the lack of CKM unitarity in the evaluation of the box diagrams, since these effects are small compared to the tree-order $Z$-mediated ones. For the sake of convenience we follow the notations of ref. [7] to write:

$$
\left|V_{t b} V_{t d}^{*}\right|^{I S}\left|\Delta_{b d}\right|^{1 / 2}=F x_{d}^{1 / 2}
$$

where

$$
F=\left[\frac{6 \pi^{2}}{G_{F}^{2} \eta M_{W}^{2} M_{B}}\right]^{1 / 2} \frac{1}{\tau_{B}^{1 / 2} B_{B}^{1 / 2} f_{B}}|\bar{E}(x)|^{-1 / 2} .
$$

The effects of new physics are contained in $\left|\Delta_{b d}\right|$ in eq.(16), which is parametrized as:

$$
\Delta_{b d}=1+r_{d} e^{i 2 \theta_{b d}}
$$

with

$$
\begin{aligned}
r_{d} & =\frac{1}{\nu|\bar{E}(x)|}\left|\frac{z_{b d}}{\left(V_{t b} V_{t d}^{*}\right)^{I S}}\right|^{2}, \quad \nu=\frac{\alpha}{4 \pi \sin ^{2} \theta_{W}}, \\
\theta_{b d} & =\arg \left[\frac{z_{b d}}{\left(V_{t b} V_{t d}^{*}\right)^{I S}}\right]
\end{aligned}
$$

In the above expressions, the experimental inputs are given by [9] $x_{d}=0.71 \pm 0.07$, $\tau_{B}=(1.54 \pm 0.03) \mathrm{ps}$, and $\sqrt{B_{B} f_{B}^{2}}$ lies between 110 and $270 \mathrm{MeV} ; \bar{E}(x)$ is the standard Inami-Lim function for the $t$-mediated box diagram and $\nu \bar{E}=-0.0065$ for $m_{t}=174$ $\mathrm{GeV}$. The phase $\theta_{b d}$ is an independent parameter, which determines the orientation of $z_{b d}$ in the unitarity quadrangle and plays an important role since it allows for different contributions of new physics even for a fixed $\left|z_{b d}\right|$. Since the aim of our analysis is to focus on the departure from the SM prediction due to unitarity violation, we fix the 
experimentally derived inputs in eqs. (16)-(19) at their central values. As a result, our estimates of the effects of new physics are conservative; one could always obtain an enhanced effect by setting the experimental inputs at their extrema.

In eq. (19) one can put $\left|V_{t b}\right|^{I S}=1$ as a good approximation. Solving for $\left|V_{t d}\right|^{I S}$ using eqs. (16) - (19) for $\theta_{b d}$ in the range $\left[0^{\circ}-180^{\circ}\right]$ for fixed values of $\left|z_{b d}\right|$ within the allowed domain as shown in eq. (11), we obtain the allowed values of $\left|V_{t d}\right|^{I S}$, displayed in fig. 2. For large values of $\left|z_{b d}\right|, \theta_{b d}$ is constrained to a given range in order for the solution for $\left|V_{t d}\right|^{I S}$ to exist. We present our results only for $m_{t}=174 \mathrm{GeV}$ and remark that they are quite insensitive to its choice in the range $m_{t}=174 \pm 17 \mathrm{GeV}$ [10]. The region between the horizontal lines corresponds to the SM uncertainties, taking into account the experimental uncertainties of the various inputs.

In fig. 3 we present the ratio $\operatorname{Br}(b \rightarrow d \gamma) / B r(b \rightarrow s \gamma)$ as a function of $\theta_{b d}$ for the same values of $z_{b d}$ as in fig. 2 and putting $z_{b s}=0$. We find that even if one allows for $z_{b s} \neq 0$, the branching ratio $\operatorname{Br}(b \rightarrow s \gamma)$ does not differ significantly from its SM value. The reason is essentially due to the fact that although $z_{b s}$ and $z_{b d}$ could be of the same order of magnitude, $\left|V_{t s}\right|$ is much larger than $\left|V_{t d}\right|$. Combining the results of the quadrangular unitarity [eq. (12)] and the information of the CKM matrix elements, it has been shown [7] that:

$$
\left|\frac{z_{b d}}{V_{t b} V_{t d}^{*}}\right| \leq 0.93 ; \quad\left|\frac{z_{b s}}{V_{t b} V_{t s}^{*}}\right| \leq 0.04 .
$$

As a result of bounds in eq. (20), one concludes that in the case of $B_{d}$ mixing, tree-level flavour-changing $Z$-exchange may give a dominant contribution, while in the case of $B_{s}$ mixing, the $t$-mediated box diagrams are the dominant ones [7, 11].

It should be noted that in the SM the ratio $\operatorname{Br}(b \rightarrow d \gamma) / B r(b \rightarrow s \gamma)$ is given, to a very good approximation, by

$$
R=\frac{B r(b \rightarrow d \gamma)}{B r(b \rightarrow s \gamma)} \simeq \frac{\left|V_{t d}\right|^{2}}{\left|V_{t s}\right|^{2}}
$$

The result of eq. (21) is quite reliable since most of the uncertainties, such as the value of $m_{b}$ and the bulk of QCD corrections, cancel out in the ratio.

It is clear from fig. 3 that as a result of violation of CKM unitarity, $R$ can significantly deviate from the SM prediction, for values of $z_{b d}$ consistent with the bound of eq. (11). Note that in fig. 3, we have indicated for the SM the regions of allowed $R$ values within the horizontal lines taking into account the experimental uncertainties of the various inputs [9], while for the present model we have taken central values for various experimental inputs. Obviously, the effect of new physics becomes more "visible" once the SM prediction is sharpened through a more precise knowledge of $V_{t d}^{S M}$ and $V_{t s}^{S M}$. 
We should, however, point out that the experimental extraction of the ratio $R$ of eq. (21) is not so straightforward on account of both QCD corrections and uncertainties in the hadronic matrix elements. For decays into light quarks, exclusive final states are theoretically "cleaner". However, in the case of $B \rightarrow X_{d} \gamma$, a double Cabibbosuppressed process, one has a situation where the $O\left(\alpha_{s}\right)$ corrections involving virtual $u$ and $c$ quarks are no longer negligible [12]. The partial width is thus no longer a simple function of $\left|V_{t d}\right|^{2}$. Apart from this, there is also the experimental problem of measuring the above inclusive decay rate. The exclusive modes are easier to measure experimentally, although they are even more difficult to handle theoretically. As an illustration, let us consider

$$
\frac{\Gamma\left(B_{u, d} \rightarrow \rho \gamma\right)}{\Gamma\left(B_{u, d} \rightarrow K^{*} \gamma\right)}=\frac{\left|V_{t d}\right|^{2}}{\left|V_{t s}\right|^{2}} \widetilde{R} \Phi_{u, d}
$$

where $\widetilde{R}=\left|F_{1}^{B \rightarrow \rho}(0)\right|^{2} /\left|F_{1}^{B \rightarrow K^{*}}(0)\right|^{2}$, the ratio of the hadronic form factors, and $\Phi_{u, d}$ is a phase space factor. While in the exact $S U(3)$ limit the hadronic uncertainties would cancel to leave $\widetilde{R}=1$, in the real world one has to calculate it within some model. An idea of the uncertainties involved can be formed by looking at the results obtained from different approaches for $\widetilde{R}=0.04$ (Isgur-Scora-Grinstein-Wise formalism), 0.6 (QCD sum rule on the light cone) and 0.8 (Bauer-Stech-Wirbel formalism) [13, 14. Another correction, in this context, has to do with the final-state interactions, which may be important for $B \rightarrow \rho \gamma$, but not so for $B \rightarrow K^{*} \gamma$ [14.

At present, no decay of the type $B \rightarrow X_{d}+\gamma$ has been observed and only upper bounds have recently been obtained by the CLEO collaboration [15] for the following exclusive decays (90\% C.L.):

$$
\begin{aligned}
\operatorname{Br}\left(B \rightarrow \rho^{-} \gamma\right) & <1.8 \times 10^{-5} \\
\operatorname{Br}\left(B \rightarrow \rho^{0} \gamma\right) & <3.1 \times 10^{-5} \\
\operatorname{Br}(B \rightarrow \omega \gamma) & <1.4 \times 10^{-5}
\end{aligned}
$$

From eq. (23) and within the SM, the following bound has been extracted [15]:

$$
\left|\frac{V_{t d}}{V_{t s}}\right|<\frac{1}{1.8}
$$

which in turn implies within the SM, using eq. (21),

$$
\frac{B r(b \rightarrow d \gamma)}{B r(b \rightarrow s \gamma)}<0.31
$$

It can be readily seen that the estimation of the bound of eq. (25) also holds in our model, and it is compatible with the prediction of our model even for the largest 
value of $\left|z_{b d}\right|$. Furthermore, it is evident that the experimental bound in eq. (23) is still not strong enough for the bound of eq. (24) to be competitive with the present SM limit on the same, derived from $B_{d}-\bar{B}_{d}$ mixing and unitarity. However, it is clear that improved data and the eventual detection of the above exclusive decays will provide an important constraint on the SM and have the potential to uncover new physics.

The presence of flavour-changing $Z$-mediated interactions has other phenomenological consequences. Recently, it has been pointed out 16 that in the presence of a singlet down-type quark, the SM prediction $7 \leq x_{s} \leq 40$ changes to $2 \leq x_{s} \leq 50$. This result is specially relevant if the experimental lower bound on $x_{s}$ turns out to be smaller than the lower bound predicted by the SM. It is clear that once the experimental value of $x_{s}$ is known and if one allows for $z_{b s} \neq 0$, the extraction of $\left|V_{t s}\right|^{I S}$ would be entirely analogous to the one we have described for $\left|V_{t d}\right|^{I S}$.

Furthermore, it has been pointed out in refs. [7, 11] that in the presence of violation of CKM unitarity, $\mathrm{CP}$ asymmetries in $B$-decays can differ significantly from those predicted in the SM. In particular, it has been shown [7] that the sign of CP asymmetry in the decay $B \rightarrow \Psi K_{s}$ may be opposite to the one predicted by the SM, even for rather small values of $z_{b d}$.

At this point the following comment is in order. It is clear that the primary task for the next generation of experiments is to check whether all data on rare $B$-decays, $B-\bar{B}$ mixings and CP asymmetries can be accommodated within the SM. If this fit is not possible, thus implying evidence for new physics, the next task would then be to discover what the new physics is and, in particular, whether there is any violation of CKM unitarity, and measuring the parameters $z_{b d}$ and $\theta_{b d}$.

To conclude, the measurement of the ratio $\operatorname{Br}(b \rightarrow d \gamma)$ together with the measurement of CP asymmetry in B-decays will provide a crucial test of the CKM unitarity, leading either to the discovery of unitarity violation, or to strong constraints on the parameters $z_{b d}$ and $\theta_{b d}$.

\section{Acknowledgements}

G.B. and G.C.B. would like to thank A. Ali for useful conversations. G.C.B thanks the CERN Theory Division for their hospitality, and T. Morozumi and L. Handoko for correspondence concerning their related work in progress. The work of G.C.B. was supported in part by Science Project No. SCI-CT91-0729 and EC contract No. CH RX - CT93 - 0132. 


\section{References}

[1] CLEO Collaboration, R. Ammar et al., Phys. Rev. Lett. 71 (1993) 674;

T. Browder, K. Honscheid and S. Playfer, in "B Decays", 2nd edition, ed. S. Stone (World Scientific, Singapore, 1994).

[2] T. Inami and C.S. Lim, Prog. Theor. Phys. 65 (1981) 297.

[3] F. Zwirner, Int. J. Mod. Phys. A3 (1988) 49;

J.L. Hewett and T.G. Rizzo, Phys. Rep. 183 (1989) 193 and references therein.

[4] L. Bento, G.C. Branco and P.A. Parada, Phys. Lett. B267 (1991) 95.

[5] A. Nelson, Phys. Lett. B136 (1984) 387;

S.M. Barr, Phys. Rev. Lett. 53 (1984) 329.

[6] R. Barbieri and G. Giudice, Phys. Lett. B309 (1993) 86;

M. Ciuchini et al., Phys. Lett. B316 (1993) 127;

M. Misiak, Phys. Lett. B269 (1991) 161.

[7] G. Branco, T. Morozumi, P.A. Parada and M.N. Rebelo, Phys. Rev. D48 (1993) 1167.

[8] UA1 Collaboration, C. Albajar et al., Phys. Lett. B262 (1991) 163.

[9] See e.g. A. Ali and D. London, preprint CERN-TH. 7248/94 (1994).

[10] CDF Collaboration, F. Abe et al., preprint FERMILAB-PUB-94/097-E (1994).

[11] Y. Nir and D. Silverman, Phys. Rev. D22 (1990) 1477;

D. Silverman, Phys. Rev. D45 (1992) 1800;

A. Ray-Mukhopadhyaya and A. Raychaudhuri, Phys. Rev. D37 (1988) 807;

B. Mukhopadhyaya, A. Ray and A. Raychaudhuri, Phys. Lett. B186 (1987) 147.

[12] A. Ali and C. Greub, Phys. Lett. B287 (1992) 191.

[13] A. Ali, V.M. Braun and H. Simma, preprint CERN-TH.7118/93, MPI-Ph/93-77, DESY 93-193.

[14] J.M. Soares, Phys. Rev. D49 (1994) 283.

[15] D. Payne, talk representing the CLEO collaboration, Beauty '94, April 1994.

[16] Y. Nir, Phys. Lett. B327 (1994) 85. 


\section{Figure captions}

1. [a] The $W$-mediated photon-penguins in the Feynman gauge. The corresponding gluon-penguins are realized by replacing the external photon lines by gluon ones in diagrams 5 and 6.

[b] The $Z$-mediated photon-penguins in the Feynman gauge. The longitudinal mode contributions are not shown, as they are negligible. The corresponding gluon-penguins are realized by replacing the external photon lines by gluon ones in all the diagrams.

2. Variation of $\left|V_{t d}\right|^{I S}$ with $\theta_{b d}$ for different values of $\left|z_{b d}\right|\left[1.16 \times 10^{-3}\right.$ (dot-dashed), $0.78 \times 10^{-3}$ (dotted) and $0.39 \times 10^{-3}$ (dashed)], evaluated using the central values of the experimental inputs $\left(x_{d}, \tau_{B}, \sqrt{B_{B} f_{B}^{2}}\right.$ etc.) and for $m_{t}=174 \mathrm{GeV}$. The region between the horizontal lines corresponds to the allowed values within the SM, taking into account the experimental uncertainties of the above inputs.

3. Variation of $R=\operatorname{Br}(b \rightarrow d \gamma) / B r(b \rightarrow s \gamma)$ with $\theta_{b d}$ for the same values of $z_{b d}$ and the same central values of the experimental inputs as in fig. 2. The horizontal band corresponds to the allowed values of $R$ within the SM for the uncertainties in various inputs as described in fig. 2 . 
This figure "fig1-1.png" is available in "png" format from: http://arxiv.org/ps/hep-ph/9406424v1 


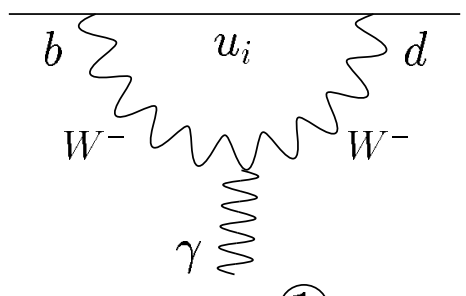

(1)

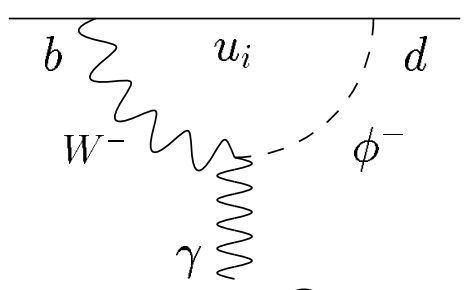

(3)

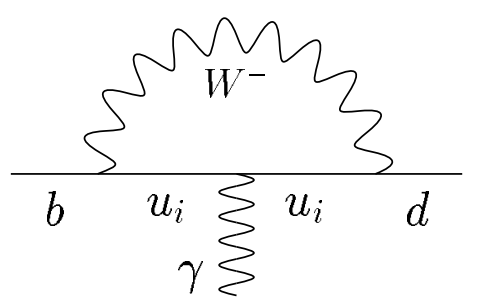

(5)

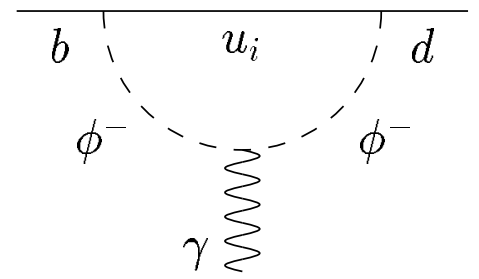

(2)

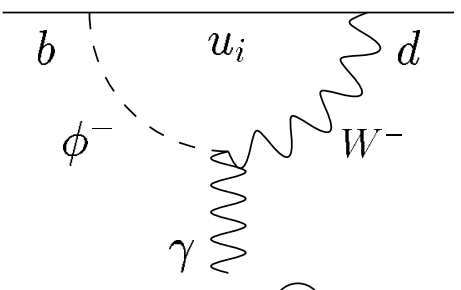

(4)

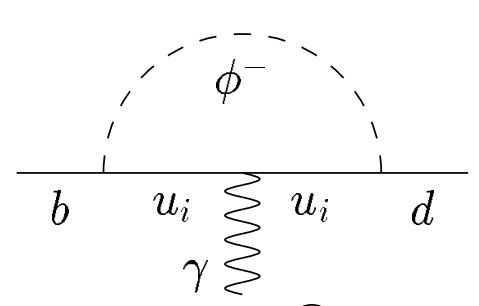

(6)

Fig. 1(a) 


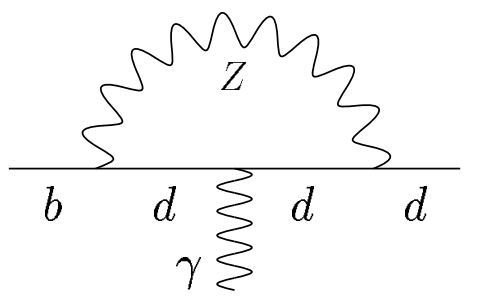

(1)

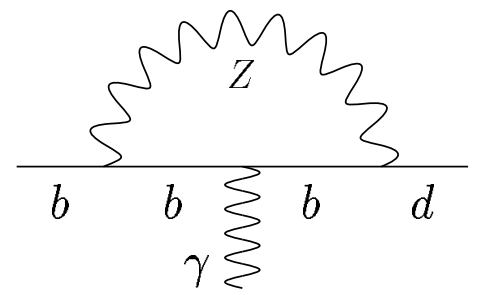

(2)

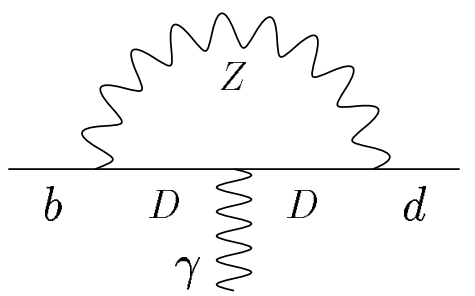

(3)

Fig. 1(b) 
This figure "fig1-2.png" is available in "png" format from: http://arxiv.org/ps/hep-ph/9406424v1 


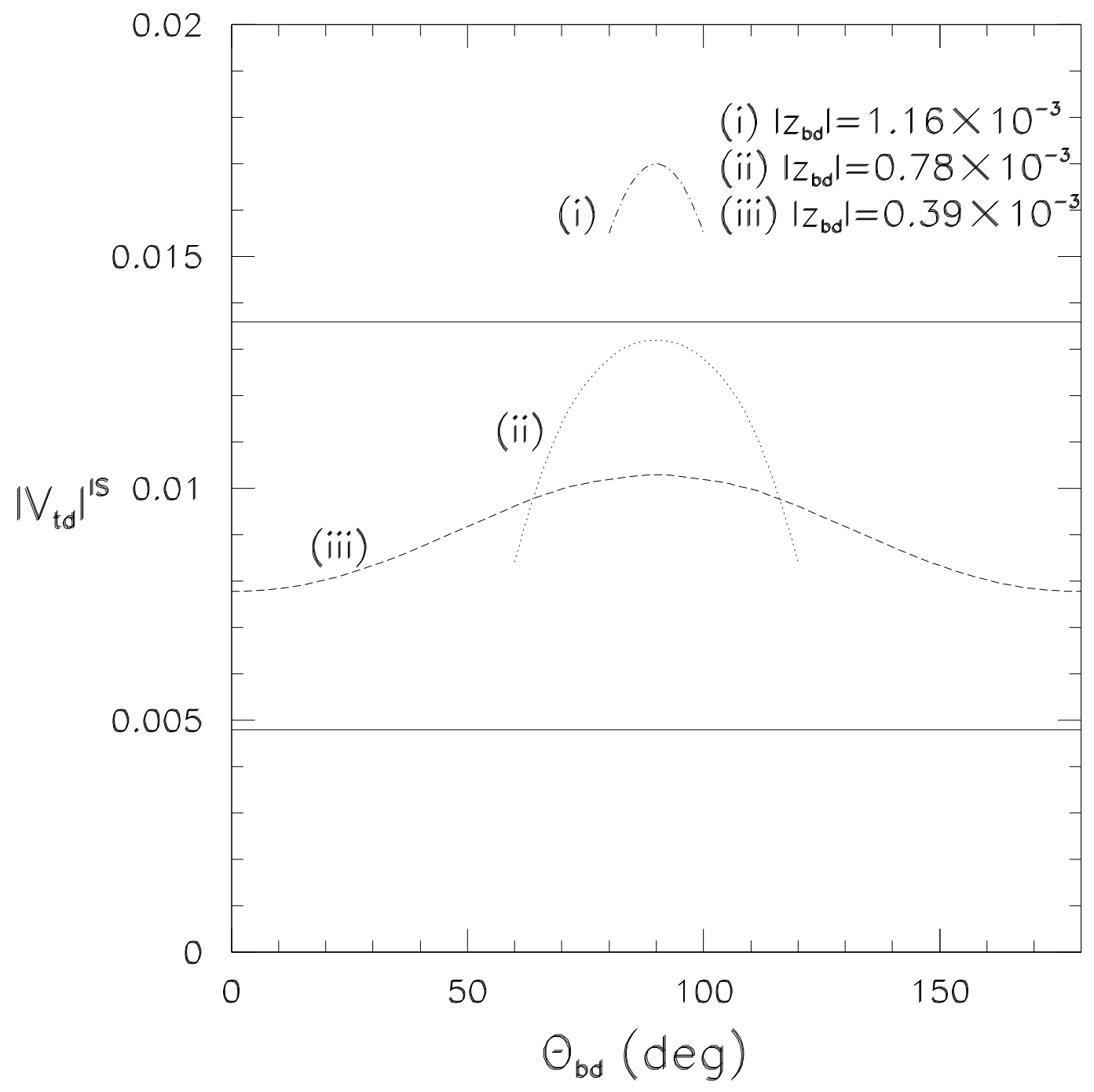

Fig. 2 
This figure "fig1-3.png" is available in "png" format from: http://arxiv.org/ps/hep-ph/9406424v1 


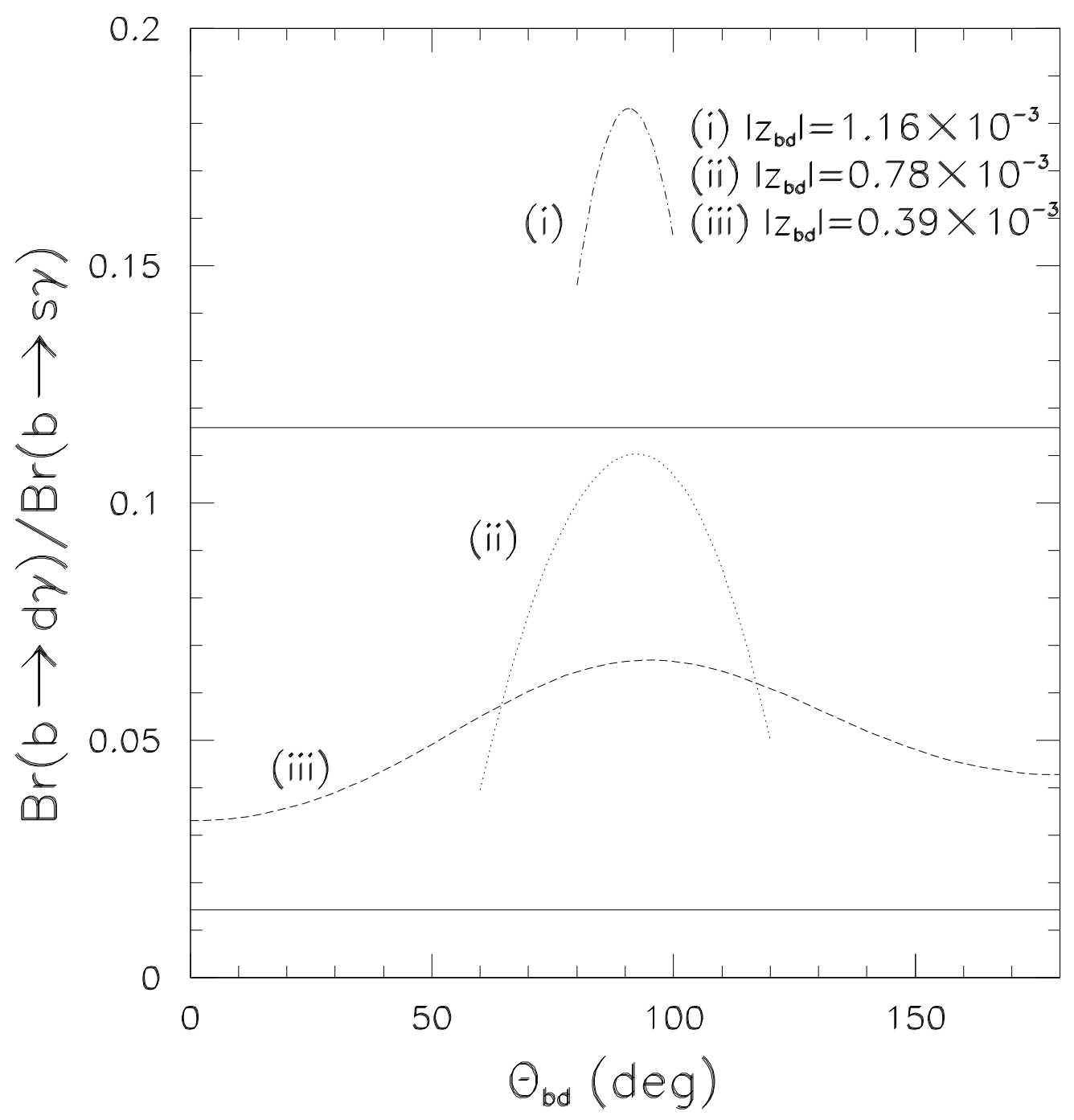

Fig. 3 